\title{
ANÁLISE EXPERIMENTAL DA DINÂMICA DE GRÃOS DE CAFÉ VERDE EM UM TAMBOR ROTATÓRIO NO REGIME DE ROLAMENTO
}

\author{
A.H. JESUS ${ }^{1}$, D. P. FURTADO ${ }^{1}$, I. A. RESENDE ${ }^{1}$, M. A. S. BARROZO ${ }^{1}$ e C. R. DUARTE ${ }^{1}$ \\ ${ }^{1}$ Universidade Federal de Uberlândia, Faculdade de Engenharia Química \\ E-mail para contato: claudiofequfu@gmail.com
}

\begin{abstract}
RESUMO - O Brasil se destaca como maior produtor, exportador e segundo maior consumidor mundial de café. Sabe-se que tambores rotatórios podem ser utilizados durante o beneficiamento do café, nas etapas de secagem e torrefação. Sendo assim, o estudo dos fenômenos físicos que ocorrem no interior de um tambor rotatório é de extrema importância para conhecer a dinâmica das partículas em seu interior. As propriedades do material particulado, as características geométricas e as condições operacionais do tambor influenciam no surgimento de diferentes regimes de escoamento das partículas. No presente estudo, com o objetivo de caracterizar o comportamento do material particulado no regime de rolamento, especificamente, utilizou-se uma técnica de análise de imagens gravadas com uma câmera de alta velocidade. Obteve-se, experimentalmente, os perfis de velocidade das partículas de café verde no regime de rolamento para diferentes níveis de enchimento e velocidades de rotação do equipamento. Sendo assim, os resultados possibilitaram uma análise da influência da velocidade de rotação do cilindro e do nível de preenchimento do tambor sobre o deslocamento das partículas e a espessura da camada ativa no interior do tambor.
\end{abstract}

\section{INTRODUÇÃO}

O café desempenha um papel de extrema importância no cenário econômico, político, social e ambiental do Brasil. O fruto é produzido em quatro continentes, sendo que as regiões que apresentam maior projeção mundial se encontram na América do Sul, África, Ásia e América Central. O Brasil é o maior produtor, exportador e o segundo maior consumidor mundial de café (Thomaziello et al., 2013; EMBRAPA, 2015).

Os estados que mais se destacam na produção de café são Minas Gerais, que é responsável por 51,59\% da produção do país; e o Espírito Santo, correspondendo a 24,76\% da produção nacional. Estudos feitos pela ABIC (Associação Brasileira da Indústria de Café) em 2016 mostram que o café é consumido em 98,2 \% dos lares brasileiros e seu consumo tem crescido nos últimos anos. Diante da grande demanda de mercado imposta sobre o produto, estudos relacionados a melhoria do processo de beneficiamento do café são de grande relevância.

Sabe-se que os tambores rotatórios possuem uma ampla aplicação em diferentes setores industriais como misturadores, granuladores, reatores, secadores, dentro outros. Em especial, 
os tambores rotatórios são utilizados durante o beneficiamento do café, nas etapas de secagem e torrefação. Segundo Ding et al. (2001) este uso generalizado pode ser explicado devido à sua capacidade de lidar com vários tipos de matérias-primas como, por exemplo, lamas e materiais granulares que apresentam uma larga faixa de distribuição de tamanhos e diferenças significativas em suas propriedades físicas.

Como observado por Mellmann et al. (2001) o movimento dos sólidos nos tambores rotatórios é dependente de diferentes variáveis operacionais e de elementos internos do tambor. De acordo com tais variáveis podem surgir no interior dos tambores rotatórios os regimes de deslizamento, caimento ou "avalanche", rolamento, cascateamento, catarateamento e centrifugação. Dentre estes, os regimes de caimento, rolamento e cascateamento, são os mais utilizados em processos industriais, como por exemplo, granulação, mistura, secagem e recobrimento, enquanto que o regime de catarateamento é, empregado nos processos de moagem (Henein et al., 1983).

No regime de rolamento, interesse do estudo desenvolvido no presente trabalho, o leito do material pode ser dividido em duas zonas distintas, sendo elas: a região passiva onde as partículas são arrastadas para cima pela parede do tambor; e a região ativa, de menor espessura, na qual as partículas se deslocam sobre a superfície do leito. Na região passiva, o material se move como um corpo rígido e a taxa de mistura entre as partículas é insignificante. Já na camada ativa, a mistura do material é intensa e ocorrem os principais mecanismos de transferência de massa e energia (Boateng e Barr, 1997; Santos et al., 2015).

Dessa forma, esse trabalho obteve os perfis de velocidades dos grãos de café verde referentes ao regime de rolamento em diferentes condições operacionais para avaliar a influência da velocidade de rotação do tambor e do seu grau de enchimento sobre a espessura da camada ativa.

\section{MATERIAIS E MÉTODOS}

O material particulado utilizado para os testes foram grãos de café verde (Figura 1). A densidade do material $\left(\rho_{S}\right)$ foi medida com um picnômetro a gás hélio (modelo Micromeritics AccuPyc 1331). O diâmetro característico $\left(\mathrm{d}_{50}\right)$ e a esfericidade dos grãos $(\phi)$ foram determinados com o equipamento CAMSIZER, que utiliza uma técnica de análise de imagens projetadas das partículas analisadas. Também obteve-se a umidade do café $(U)$, em base seca, pelo método da estufa por $24 \mathrm{~h}$ a $105^{\circ} \mathrm{C}$. Os valores encontrados para estas propriedades estão listados na Tabela 1.

Figura 1 - Grãos de café verde

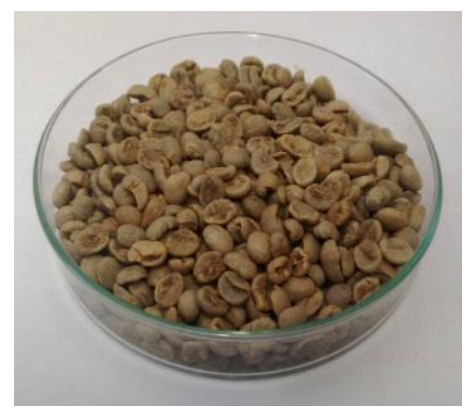


Tabela 1 - Propriedades do café verde

\begin{tabular}{|cccc|}
\hline $\mathbf{\rho s}\left[\mathbf{k g} / \mathbf{m}^{3}\right]$ & $\mathbf{d} \mathbf{5 0}[\mathbf{m m}]$ & $\boldsymbol{\phi}[-]$ & $\mathbf{U}[-]$ \\
\hline $1368,3 \pm 3,9$ & 5,24 & 0,88 & $12,15 \pm 0,10$ \\
\hline
\end{tabular}

A unidade experimental empregada para realização deste estudo está indicada na Figura 2. Esta unidade possui um tambor de aço inox (item 1) de dimensões: diâmetro de $21,5 \mathrm{~cm}$ e comprimento igual a $50 \mathrm{~cm}$, um motor (item 2) de $1710 \mathrm{rpm}$ e um inversor de frequência (item 3), para controlar a velocidade de rotação do tambor. As paredes frontal e traseira do tambor são constituídas de vidro para possibilitar a visualização do interior do equipamento e facilitar a filmagem do material em escoamento, feita com uma câmera de alta velocidade (item 4) (velocidade máxima de captura de 2000 frames/s). O interior do tambor é revestido por uma lixa P80 para evitar o regime de deslizamento e possibilitar a formação dos regimes de interesse mesmo nos menores níveis de enchimento.

Os perfis de velocidade das partículas foram obtidos por meio das filmagens feitas com a câmera de alta velocidade e a utilização dos softwares ImageJ e Meazure ${ }^{T M}$, possibilitando o mapeamento individual das partículas em diferentes quadros. A velocidade de cada uma das partículas foi calculada pela subtração da posição inicial e final e posterior divisão pelo intervalo de tempo. A Figura 3 apresenta a localização da linha de referência para o raio do leito, ao longo da qual foram medidos os valores de velocidade. Para cada raio (r), as medidas foram feitas em duplicatas.

Figura 2 - Unidade experimental e a câmera de alta velocidade utilizadas nos experimentos

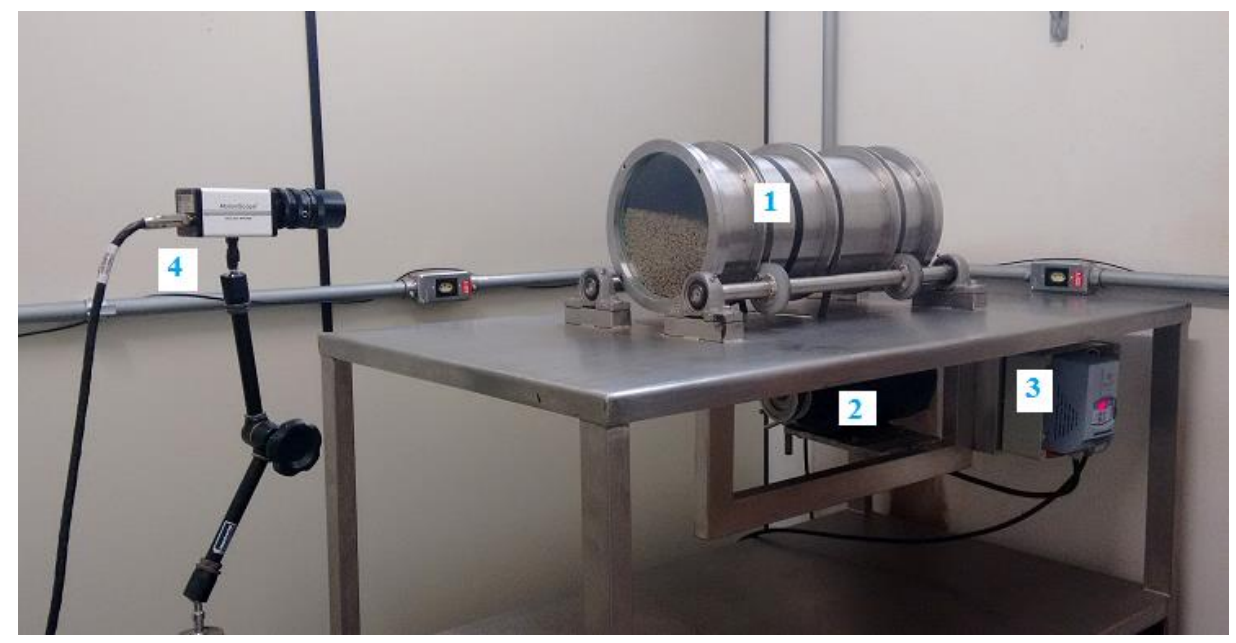

Para os 5 níveis de enchimentos avaliados (10, 20, 30, 40 e 50\%) as velocidades de rotação do tambor utilizadas para obtenção dos perfis de velocidades foram: 12, 13, 16 e 18 rpm.

Para determinar a localização da interface ativa-passiva (região da inversão de sentido das velocidades), foi observado que tanto na camada ativa quanto na camada passiva, a velocidade varia linearmente com o raio. Assim, as equações das retas para descrever cada 
uma das regiões foram encontradas e então, a interseção entre as duas retas determinava o ponto de transição entre as camadas.

Figura 3 - Região ativa, região passiva e linha de referência usada na metodologia para calcular os perfis de velocidade das partículas

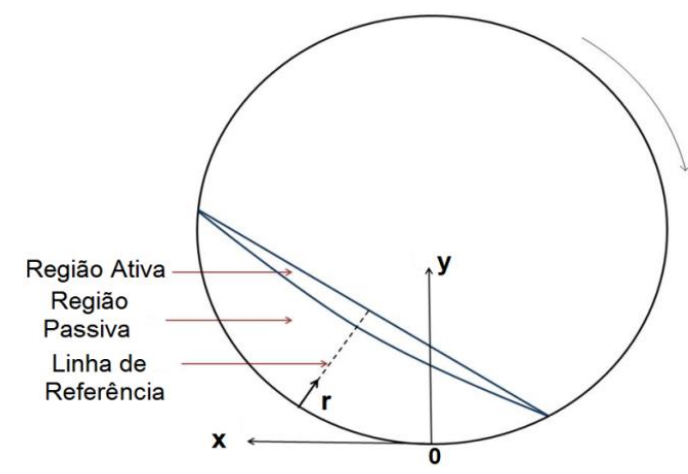

\section{RESULTADOS}

Para determinação da espessura da camada ativa, região do leito de partículas observada no regime de rolamento na qual ocorre a mistura efetiva do material particulado, foram obtidos inicialmente os perfis de velocidade dos grãos de café verde para diferentes condições operacionais.

A Figura 4 apresenta perfis de velocidade típicos para o material particulado em estudo. Tais dados foram obtidos para um o grau de enchimento constante e igual a $10 \%$ para as velocidades de rotação do tambor iguais a 12,13, 16 e $18 \mathrm{rpm}$. Todos os perfis de velocidade apresentados na Figura 4 possuem um comportamento característico, que também foi observado para todas as demais condições avaliadas. Nota-se que para a posição mais próxima da parede do tambor, as partículas apresentaram velocidades próximas a velocidade de rotação do tambor. Com o aumento da posição, esta velocidade decresce até próxima de zero, neste ponto tem-se a inversão do sentido de deslocamento do material e o final da camada passiva. Após o ponto de inversão, localiza-se a região ativa que é caracterizada pelo aumento da velocidade à medida que aumenta-se a posição.

Na Tabela 2 estão apresentados os valores de espessura da camada ativa para as condições experimentais empregadas. Em ordem crescente do grau de enchimento do cilindro, tem-se que da menor rotação testada $(12,0 \mathrm{rpm})$ para a maior $(18,0 \mathrm{rpm})$ há um aumento nesta espessura de: 20, 25\% (enchimento de 10\%), 13, 55\% (enchimento de 20\%), 28,70\% (enchimento de 30\%), 25,51\% (enchimento de 40\%) e 16,38\% (enchimento de 50\%). Nota-se então, que o aumento da velocidade de rotação do tambor leva a um aumento da espessura da região ativa.

Além disso, mantendo-se a velocidade de rotação do tambor a $12 \mathrm{rpm}$ e variando-se o enchimento de $10 \%$ para $50 \%$, observa-se um aumento da espessura da região ativa igual a $79,75 \%$. Considerando-se essa mesma variação no enchimento, tem-se um aumento da espessura de 73,41\% (velocidade de $13 \mathrm{rpm}$ ), 77,47\% (velocidade de $16 \mathrm{rpm}$ ) e 73,97\% (velocidade de $18 \mathrm{rpm}$ ). Pode-se afirmar, assim, que um aumento no nível de enchimento do 
tambor rotatório tem como consequência o aumento da espessura da camada ativa no regime de rolamento.

Figura 4 - Perfil de velocidade dos grãos de café verde para as velocidades de rotação do tambor: (a) $12 \mathrm{rpm}$; (b) $13 \mathrm{rpm}$; (c) $16 \mathrm{rpm}$; (d) $18 \mathrm{rpm}$.

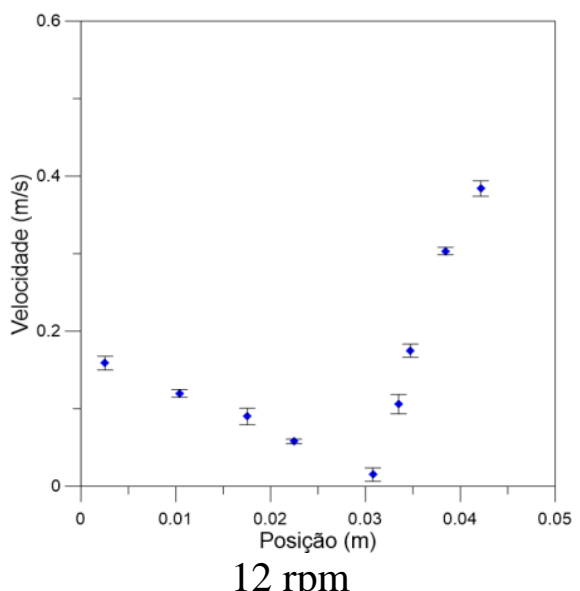

(a)

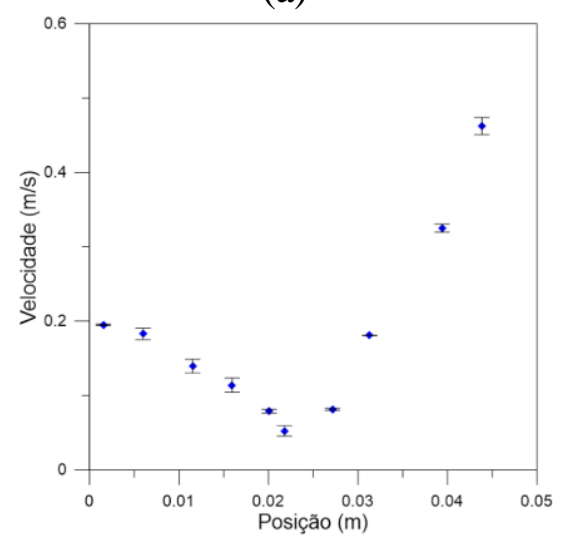

$16 \mathrm{rpm}$

(c)

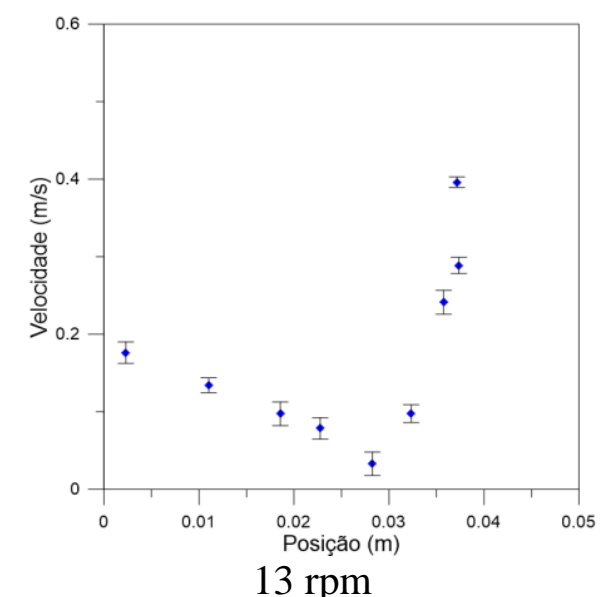

(b)

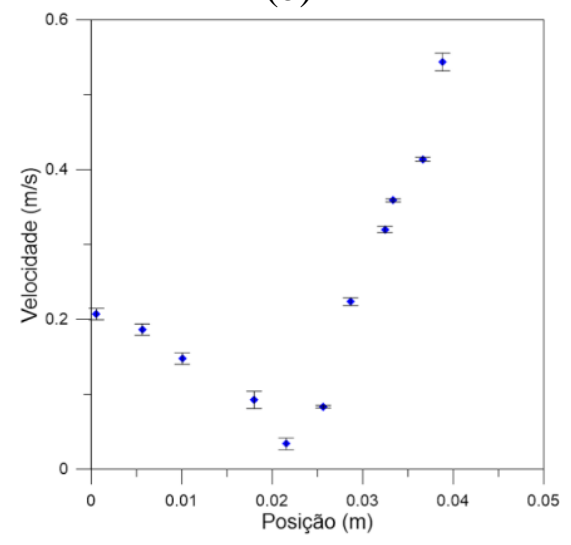

$18 \mathrm{rpm}$

(d)

Tabela 2 - Variação da espessura da camada ativa com o enchimento e velocidade de rotação do tambor para o café verde.

\begin{tabular}{|c|ccccc|}
\hline \multirow{2}{*}{$\begin{array}{c}\text { Velocidade do } \\
\text { Tambor (RPM) }\end{array}$} & \multicolumn{5}{|c|}{ Espessura da Camada Ativa (cm) } \\
\cline { 2 - 6 } & $\mathbf{1 0 \%}$ & $\mathbf{2 0 \%}$ & $\mathbf{3 0 \%}$ & $\mathbf{4 0 \%}$ & $\mathbf{5 0 \%}$ \\
\cline { 2 - 6 } & $1,63 \pm 0,02$ & $2,14 \pm 0,01$ & $2,16 \pm 0,03$ & $2,43 \pm 0,03$ & $2,93 \pm 0,13$ \\
$\mathbf{1 2 , 0}$ & $1,73 \pm 0,02$ & $2,34 \pm 0,06$ & $2,51 \pm 0,05$ & $2,63 \pm 0,05$ & $3,00 \pm 0,09$ \\
$\mathbf{1 3 , 0}$ & $1,82 \pm 0,08$ & $2,35 \pm 0,03$ & $2,60 \pm 0,03$ & $2,81 \pm 0,03$ & $3,23 \pm 0,04$ \\
$\mathbf{1 6 , 0}$ & $1,96 \pm 0,13$ & $2,43 \pm 0,10$ & $2,78 \pm 0,08$ & $3,05 \pm 0,08$ & $3,41 \pm 0,04$ \\
$\mathbf{1 8 , 0}$ & & & & & \\
\hline
\end{tabular}




\section{CONCLUSÃO}

O estudo experimental desenvolvido no presente trabalho permitiu a análise, de maneira mais detalhada, da dinâmica dos grãos de café no interior de um tambor rotatório no regime de rolamento. A técnica de imagens empregada para determinar os perfis de velocidade do material particulado utilizado no estudo obteve resultados satisfatórios. Por meio da metodologia usada também foi possível encontrar a espessura da camada ativa relativa as diferentes velocidades de rotação $(12,13,16$ e $18 \mathrm{rpm})$ e graus de enchimento do cilindro (10, $20,30,40$ e $50 \%$ ) testados.

Tendo em vista os resultados experimentais apresentados, foi possível concluir que a espessura da camada ativa é fortemente influenciada pelo nível de enchimento e também pela rotação do tambor. A caracterização desta região do leito é relevante uma vez que nela ocorrem os principais mecanismos de transferência de massa e energia.

\section{REFERÊNCIAS}

ABIC, Consumo de café no Brasil mantém-se quase estável e acima de 20 milhões de sacas. Disponível em: http://www.abic.com.br/publique/cgi/cgilua.exe/sys/start.htm?sid=61\#consint2015.2, Acessado em 03/11/16.

BOATENG, A.A., BARR, P.V., Granular flow behaviour in the transverse plane of a partially filled rotating cylinder, Journal of Fluid Mechanics, v. 330, p. 233-249, 1997.

DELELE, M.A., WEIGLER, F., FRANKE, G., MELLMANN, J. Studying the solids and fluid flow behavior in rotary drums based on a multiphase CFD model. Powder Technology, vol.292, p.260-271, 2016.

DING.Y.L., SEVILLE, J.P.K., FORSTER, R.,PARKE R,D.J. Solids motion in rolling mode rotating drums operated at low to medium rotational speeds, Chemical Engineering Science, v. 56, p.1769-1780, 2001.

EMBRAPA, Café é a segunda bebida mais consumida no Brasil. Disponível em: https://www.embrapa.br/busca-de-noticias/-/noticia/2574254/cafe-e-a-segunda-bebidamais-consumida-no-brasil/, Acessado em 05/09/15.

HENEIN, H., BRIMACOMBE, J.K., WATKINSON, A.P. Experimental study of transverse bed motion in rotary kilns. Metallurgical Transactions B, v.14, p.191-205, 1983.

MELLMANN, J., The transverse motion of solids in rotating cylinders-forms of motion and transition behavior, Powder Technology, vol. 118, p. 251-270, 2001.

SANTOS, D.A., DADALTO, F.O., SCATENA, R., DUARTE, C.R., BARROZO, M.A.S., A hydrodynamic analysis of a rotating drum operating in the rolling regime, Chemical Engineering Research and Design, vol. 94, p. 204-2012, 2015.

THOMAZIELlo, R. A. Cultura do Café. 2. ed. Boletim técnico, CATI/ Campinas, n. 193, 2013. 